\title{
On the possible ultrasonic inspection of micro-bubbles generated by the optical fiber tip
}

\author{
V. V. Kazakov*, A. G. Sanin and V. A. Kamensky \\ Institute of Applied Physics of the Russian Academy of Sciences \\ 46 Ul'yanov Street, 603950, Nizhny Novgorod, Russia \\ *kazak@appl.sci-nnov.ru
}

Received 4 September 2015

Accepted 26 October 2015

Published 24 December 2015

\begin{abstract}
We demonstrate the possibility of detection and monitoring of bubbles emerging near the tip of an optical fiber by means of ultrasonic method. The excitation of bubbles at their resonant frequencies is performed using short ultrasonic pulses having a wide frequency range simultaneously with their modulation by means of a long pulse of a monochromatic frequency. This method allows detection of bubbles of various sizes. Used signal processing method, which allows increased bubble detection accuracy, is proposed for research in environments of biological-like medium which show continuous variations in structure and properties when exposed to optical emission. The method has been demonstrated on model objects: in a liquid and in a biological tissue phantom using various methods of bubble generation (hydrolysis and optical emission). We studied bubble formation by the tip of a fiber of the surgical laser LSP-007/10 "IRE Polus" with a wavelength of $0.97 \mu \mathrm{m}$ coated with a highly absorbing graphite layer.
\end{abstract}

Keywords: Bubble generation; ultrasonic inspection of bubbles; subharmonic ultrasound modulation; laser surgery.

\section{Introduction}

The laser with optical fiber having a strongly absorbing coating applied on its tip is widely used for the ablation of pathogenic biological tissues. ${ }^{1-3}$ Heating of the tip coating to high temperatures makes it possible to obtain a variety of practically significant results. On the one hand, it considerably increases the cutting speed and reduces blood loss, which has a beneficial effect on post-surgery wound healing. ${ }^{4-6}$ On the other hand, it gives rise to acoustic flows and leads to the generation of bubbles of different sizes ranging from fractions of a millimeter, the noise of which is heard as a "boiling" noise, to the size of the micron level. ${ }^{3,7,8}$ The cavitation bubbles collapse, creating powerful acoustic streaming and shock waves causing, in particular, the death of bacteria, and thereby sterilizing the wound..$^{9,10}$

*Corresponding author.

This is an Open Access article published by World Scientific Publishing Company. It is distributed under the terms of the Creative Commons Attribution 4.0 (CC-BY) License. Further distribution of this work is permitted, provided the original work is properly cited. 
Changes in tissue properties may result in nonuniform ablation followed by overheating of particular areas of the tissue and the formation of necrosis. In order to eliminate this negative factor, automatic adjustment of laser power by measuring the temperature of the optical fiber tip is used. ${ }^{2}$ Optical methods are commonly employed for detection of single bubbles at the tip of the optical fiber. This is acceptable, however, only for relatively large bubbles or bubbles moving with low speed. Multiple bubbles, including micron-sized ones, are very difficult to detect. Thus, there is a need to develop an acoustic method for bubble monitoring that would permit to use the measured results not only for ascertaining the presence of bubbles of a certain size, but also to allow some control of bubble generation similarly to the adjustment by temperature.

The most suitable solution for the above problems is the use of combination of linear and nonlinear pulse ultrasonic methods. ${ }^{7-11}$ These methods have been developed to a considerable extent for the detection of single bubbles excited by acoustic waves at their resonant frequencies of different modes of oscillation in a water. ${ }^{12}$ Use of the above methods is well-known in relation to the detection of bubbles during decompression, or phospholipidcoated micro-bubbles in order to increase ultrasonic image contrast. ${ }^{13,14}$ The peculiarity of measurement conditions at this paper is primarily that the environmental parameters and structures exposed to the optical emission and emerging acoustic flows are constantly changing. Secondly, the emerging bubbles have a wide range of sizes: from a few microns, which are prone to cavitation, to ones which are 100s of microns across and which can be observed visually.

The purpose of this work was to develop the ultrasound methods to detect the size of bubbles in a water or biological tissue generated by laser radiation near the tip of an optic fiber.

\section{Materials and Methods}

\subsection{Experimental setup}

The complex nature of bubble generation and their lifetime depends on a variety of significant factors: the bubble size, their environment, the temperature, pressure, etc. ${ }^{15-17}$ The same bubble may simultaneously have both linear and nonlinear properties, and may be excited both at its fundamental resonant frequencies, and at its ultra- and sub-harmonic frequencies. ${ }^{18-22}$ The size of a bubble changes continuously throughout its whole lifetime, and this naturally leads to corresponding changes in its resonant frequencies. Bubbles of a given size are generally detected using ultrasound location methods based on recording the amplitude of the subharmonic of the emitted ultrasonic wave. ${ }^{23-30}$ Parametric excitation of a bubble at its resonant frequency $f_{2}=f_{1} / 2$, where $f_{1}$ is the frequency of the emitted wave, allows detection of the bubble with a high degree of confidence. At the same time, bubbles will be excited on the $f_{1}$ frequency as well. Methods of excitation using short pulses are therefore preferable for the inspection of bubbles which have a range of sizes. A wide excitation spectrum allows excitation of all sizes of bubbles at the same time. The bubbles may then be categorized by size using a software-based method, by means of signal analysis in various frequency ranges. ${ }^{19}$ This work combines both methods: the excitation is performed by a rectangular radio pulse including $8-10$ cycles at the $f_{1}$ frequency. Such a pulse has steep rising edges and a pulse duty factor equal to $3-4$. This type of complex signal has a wide excitation spectrum with a predominance of excitation in synchronous mode at the $f_{1}$ and $f_{2}$ frequencies. The frequency used for determining bubble location is selected within a range up to $10 \mathrm{MHz}$, which is necessary in order to detect the micron-size bubbles that, being prone to cavitation, usefully provide an antiseptic effect.

The base diagram of the setup used for measurement is shown in Fig. 1. The layout of the transducers for different measurement conditions is shown in Fig. 2.

Initially used ultrasonic locator emitted 10 cycles at a frequency $f_{1}=2.92 \mathrm{MHz}$ in the trigger excitation mode using a rectangular pulse with pulse duty factor 3 using a $T 1$ transducer. The ultrasonic repetition pulse frequency was $f_{r}=5 \mathrm{~Hz}$, and the excitation amplitude was $40 \mathrm{~V}$. The axes of the wide-band emitting and the receiving $T 2$ transducers intersected at a distance of $d_{1}=15-20 \mathrm{~mm}$ from the plane of the working surfaces. Both transducers were $6 \mathrm{~mm}$ in diameter, and $20 \mathrm{~mm}$ long. The ultrasonic signal received from $T 2$ was amplified by a two-stage wide-band, low-noise amplifier based on AD811, and AD8005 chips, and after that sent to the first input of an AKIP-75244B USB-oscilloscope and recorded on a computer. The 


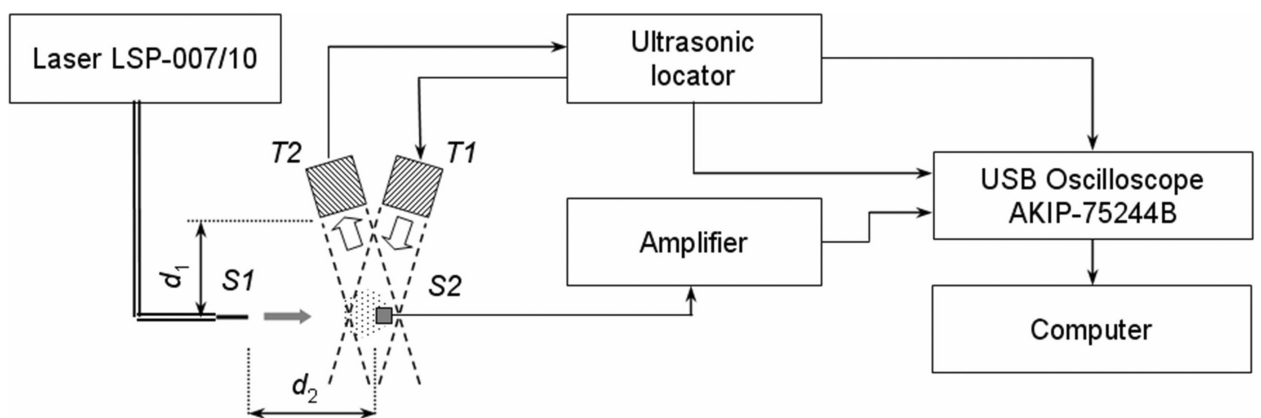

Fig. 1. Experimental setup for detection of bubbles.

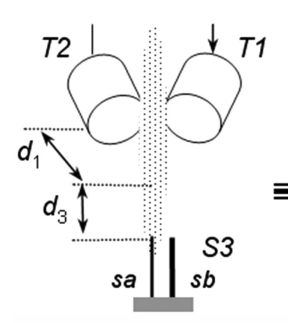

(a)

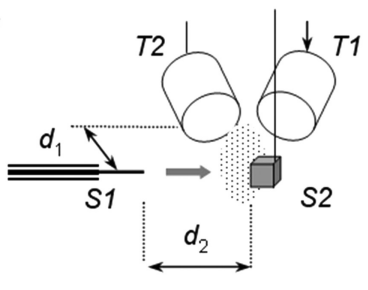

(b)

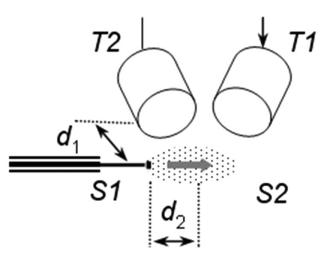

(c)

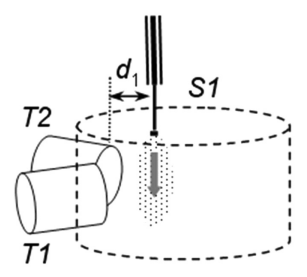

(d)

Fig. 2. Setup of transducers for various environments.

second input of the oscilloscope was used for temperature measurement. Temperature was measured with a $3.05 \times 1.4 \times 1.02 \mathrm{~mm}$ TC1047A using a standard connection to an amplifier based on an OP07CP chip.

The light source was generated by an LSP-007/ 10 "IRE Polus" surgical laser, as used for dissection and coagulation of biological tissues during surgery. The laser was operated in continuous emission mode: with a wavelength of $0.97 \mu \mathrm{m}$; a maximum power of $P=10 \mathrm{~W}$ and a silica optical fiber with a waveguide diameter of $0.5 \mathrm{~mm}$. The optical fiber tip was placed at the distances of $d_{2}=0-20 \mathrm{~mm}$, $d_{3}=0-20 \mathrm{~mm}$ from the intersection point of the $T 1$ and $T 2$ ultrasonic transducer axes.

\subsection{Measurement technique}

Processing of the signal from the ultrasonic locator output was performed as follows. Each front of signal repetition frequency actuating the emission of an ultrasound pulse started the recording of the signal reflected from the location zone using an USB-oscillograph with a 15 bit capacity and a sampling frequency of $125 \mathrm{MHz}$. Each measurement cycle, including the on/off turning of the laser, allowed the recording of up to 100 oscillograms of received signals. The same $8.2 \mu \mathrm{s}$ time interval was selected for analysis of all the recorded oscillograms, corresponded to area of bubbles occurrences. Spectra corresponding to the oscillation frequencies of the bubbles excited by the ultrasonic pulses were calculated for each oscillogram using 8192 counts.

Precise calculation of bubble sizes and their oscillation time taking into account the effects of thermal and mass transfer, changes of the surface tension coefficient and other effects depending on wavelength of ultrasound, is rather complicated, needs a lot of factors to be considered and may be found in Refs. 31-33. Since for our study the calculation accuracy was not crucial, and it was rather important to estimate only the range of sizes of the bubbles under study, the bubble radius was calculated using a simplified equation:

$$
f=\frac{\sqrt{\frac{3 \gamma P_{0}}{\rho}+\frac{(3 \gamma-1) \cdot 2 \sigma}{\rho R}}}{2 \pi R},
$$

where $\gamma=4 / 3$ is the adiabatic index of the gas, $P_{0}$ is the static pressure in the water, $\sigma$ is the surface tension, $\rho$ is the density of the water; and $R$ is the bubble radius. ${ }^{22,34,35}$

The received echo-signal typically contains multiple reflections from objects in the location zone, in 
particular, from environmental structures with different acoustic impedances, and from the tip of fiber. Those reflections mask the signals reflected by the bubbles themselves, and qualitatively distort their spectra. This necessitates a change in the processing algorithm to increase the specificity of bubble detection, a supporting signal can be subtracted from the received echo-signal. This may be done using various methods depending on the measurement conditions.

If the measurement conditions related to changes in the particular environmental parameters before and after exposure to the optical radiation do not change significantly, e.g., if the optical fiber is in water, then the supporting signal may be represented by the oscillogram recorded before the start of laser radiation. This supporting signal is subtracted from all subsequent oscillograms. Prior to substruction it is assumed that such oscillograms correspond to reflections only from the bubbles. If bubbles with different sizes independently manage to disappear or collapse within the time interval between two subsequent ultrasonic pulses, which often happens in actual practice, then the difference between the adjacent pulse signals is also insignificant, as in the previous case. If bubbles are generated continuously for each pulse, then the difference between the signals will be reduced, although bubbles will still be present. The question that has to be answered apart from this is how we can monitor the dynamic changes in the spectrum. Using the accumulation of the peak values of spectrum amplitudes reduces the noise immunity although it allows us not to miss the generation of bubbles of a particular size. Using the accumulation of average values makes the detection of individual bubbles impossible, but in this case we can identify continuously emerging bubbles in the region under study.

Choosing the processing method requires preliminary clarification both of the features of bubble generation in relation to the environment of the region under study, and the resolution of the problem of the detection of specific bubbles which it is critical not to miss. For instance, for the detection of bubbles in the blood during decompression, or for control of the overall level of bubble distribution generated in the environment, which is important for controlling laser ablation. Let us now consider the peculiarities of bubble detection in various environments used during ultrasonic inspection.

\subsection{Bubble detection in water during their generation by hydrolysis}

Verification of the proposed method of measurement was performed by means of the traditional way of detection of bubbles emerging in water by hydrolysis (Fig. 2(a)). The hydrolysis device comprised a steel needle of diameter $0.3 \mathrm{~mm}$, and $50 \mathrm{~mm}$ long, and a copper rod of diameter $1.5 \mathrm{~mm}$. There was a $3 \mathrm{~mm}$ gap between the needle and the rod. Bubbles emerged along a $15 \mathrm{~mm}$ uncoated section of the needle as a result of applying a voltage of $4 \mathrm{~V}$ DC to the rod. To initiate the generation of predominantly small bubbles, the needle was excited contactless, using a solenoid with a frequency of $39 \mathrm{~Hz}$ which matched its first flexural mode. The needle was placed below the ultrasonic location area in such a way that the jet of emerging bubbles was in a location zone of $d_{1}=20 \mathrm{~mm}$ and $d_{2}=15 \mathrm{~mm}$. The voltage was applied to the needle $t_{0}=4 \mathrm{~s}$ after starting recording. The resulting spectrum changes with time are shown in Figs. 3(a) and 3(b). Figure 3(c) shows the peak $S p(f)$ and average $S s(f)$ spectrum values in three oscillograms starting from the moment $t_{1}=3.6 \mathrm{~s}$ (noise) and $t_{2}=8 \mathrm{~s}$ (signal from the bubbles) plotted on one axis, and the calculated bubble radius values plotted on the other axis. The oscillogram for $t_{1}$ was used as the supporting signal to be subtracted.

The technique which has been developed actually allows monitoring of the generation of bubbles with sizes varying from a few millimeters to tens of millimeters, by means of linear and nonlinear (using the harmonics of the $f_{2}$ frequency) methods at the same time. If signal analysis is used in combination with subtraction of the reference signal, then the signal to noise level may reach $40 \mathrm{~dB}$ at some frequencies, greatly improving measurement accuracy. Accumulation of peak or average spectral component amplitude values shows that the changes in the spectrum envelope match qualitatively. That the ranges of signal/noise ratios of these remain the unchanged indicates that bubbles of the same size are generated continuously, but that there are also many bubbles with radius of a few microns, i.e., ones that are prone to cavitation.

\subsection{Bubble detection in water during heating by optical radiation}

Figure 2(b) shows a diagram of the setup for the detection of bubbles generated as a result of solid 


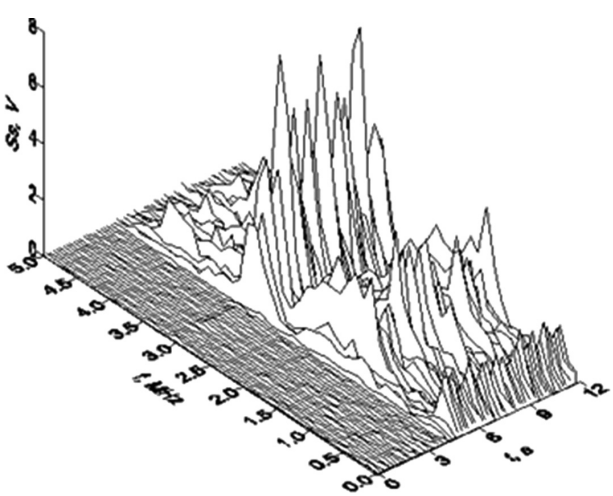

(a)

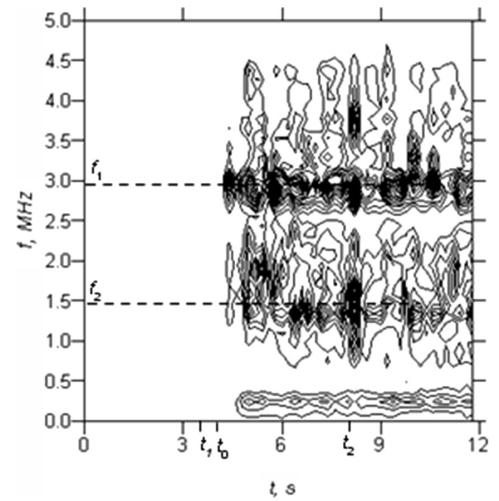

(b)

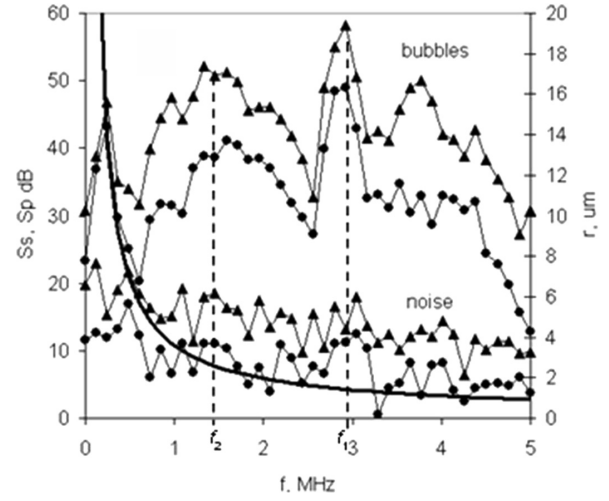

(c)

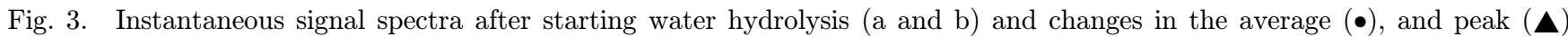
spectrum values, and bubble sizes (-) depending on frequency.

body heating in water at the same time as its temperature is measured. Optical emission was used to heat a TC1047A (S2) thermal sensor located at a distance, $d_{2}$, ranging from 2 to $20 \mathrm{~mm}$. The laser emission power $P$ was either $3 \mathrm{~W}, 6 \mathrm{~W}$, or $9 \mathrm{~W}$. Figure 4(a) demonstrates the changes in the amplitudes $U_{1}$, and $U_{2}$ of the spectral components at frequencies $f_{1}$ and $f_{2}$ as well as the average temperature of the sensor. Figure $4(\mathrm{c})$ shows the signal spectra for $P=9 \mathrm{~W}, d_{2}=4 \mathrm{~mm}, t_{1}=4 \mathrm{~s}$, $t_{0}=5 \mathrm{~s}$ (the moment of laser actuation), $t_{2}=10 \mathrm{~s}$, and $t_{3}=12 \mathrm{~s}$ (the time when the laser was turn off).

The average sensor temperature may exceed $100^{\circ} \mathrm{C}$ when $P>6 \mathrm{~W}$ and $d_{2}<6 \mathrm{~mm}$, which, being the boiling point of water, results in intensive bubble formation. The bubble generation process starts only when the laser turns on, which is confirmed by the increasing amplitudes of the signal spectral components received within the range of $0.1-$ $5.0 \mathrm{MHz}$ (see Fig. 4(a)). The intensity of bubble generation is reasonably high because the levels of the spectrum peak and the average value differ by less than $5 \mathrm{~dB}$ across the whole range (Fig. 4(b)). This difference is considerably greater during hydrolysis (Fig. 3(c)).

It is obvious that if any object reaches boiling point as a result of absorption of the optical radiation this will result in bubble generation. So strongly absorbing coating is applied on the tip of the optical fiber to increase its temperature. ${ }^{2,6,8}$ Typically, a carbon-containing compound supplied with the surgical laser is used.

However, its life time is then only tens of seconds when used for ablation in a biological environment. An increase in working time may be achieved by applying the strongly absorbing coating, made of

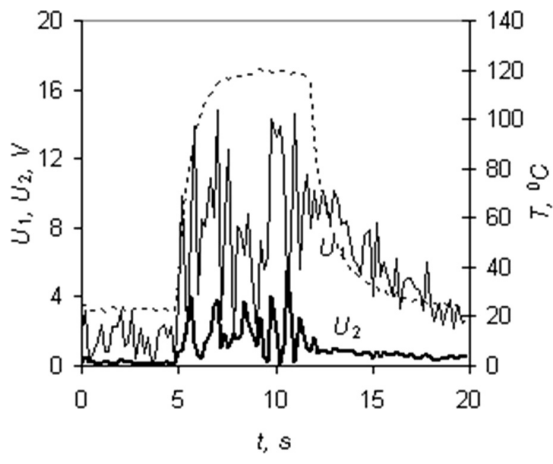

(a)

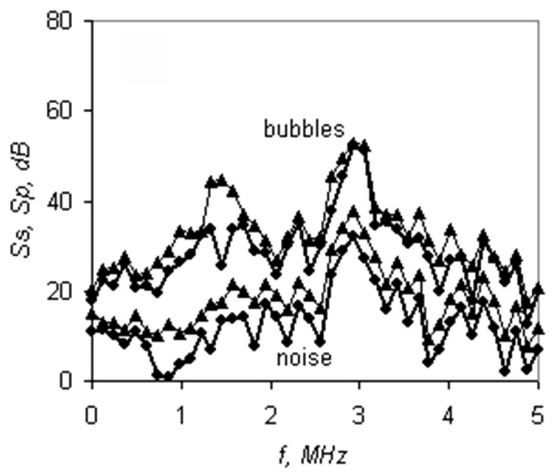

(b)

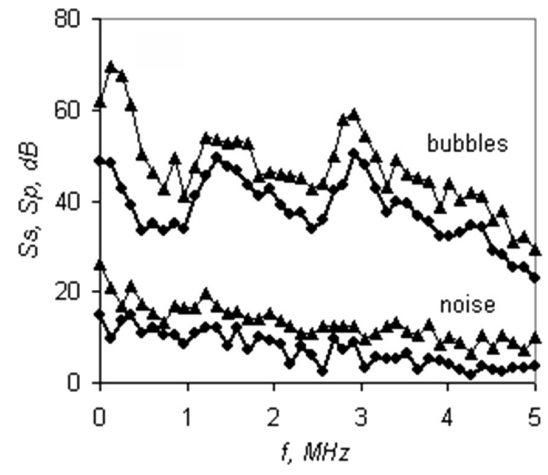

(c)

Fig. 4. Amplitude of the $f_{1}$ and $f_{2}$ frequencies and sensor temperature $(-)$, and changes of the average $(\bullet)$ and peak $(\boldsymbol{\Delta})$ values of the signal and noise spectra for un-coated optical fibers (a,b) and with a strongly absorption coating (c). 
mixture of polymethyphenylsiloxane resin filled with $0.5-1.5 \mu \mathrm{m}$ graphite powder. This specially developed technique allows the cutting of biological tissue for 10-15 min. In our experiments the extent of shading by the absorption layer did not exceed $20 \%$ of laser radiation. The setup of transducers for this case are shown in Fig. 2(c) for $d_{1}=8 \mathrm{~mm}$ and $d_{2}=12 \mathrm{~mm}$. Figure 4 (c) demonstrates the spectra of signals received during bubble generation at the heated area of water nearly to the tip of fiber. It can be clearly seen that, even in clear water, the heated optical fiber generates bubbles intensively. The level of the spectral components over noise exceeds $20 \mathrm{~dB}$ which is comparable to the level from bubbles generated during hydrolysis (see Fig. 3(c)). The coated optical fiber tip effectively generates bubbles with sizes varying from a few microns to tens of microns. Additional tests showed that a localized acoustic flow with a cross-section of less than $2 \mathrm{~mm}$ and a length of $3-5 \mathrm{~mm}$ is created along the axis of the coated optical fiber.

\subsection{Bubble detection in a phantom of tissue}

An optical fiber with a coating based on epoxy resin was used for the destruction of a tissue phantom made of $2 \%$ agar. The phantom had a cylindrical shape of $68 \mathrm{~mm}$ diameter by $28 \mathrm{~mm}$ thick (see Fig. 2 (d)). Its side surface was in contact with $T 1$ and $T 2$ transducers at a distance of $14 \mathrm{~mm}$ from the base of the cylinder. The tip of the optical fiber was pressed into the cylinder surface to a depth of $2 \mathrm{~mm}$ at the point located at the distance of $15 \mathrm{~mm}$ from the top of the cylinder. Laser heating was actuated at $t_{0}=5 \mathrm{~s}$ after starting the record of oscillograms. This resulted in a rapid melting of the agar in a direction perpendicular to the axis of the $T 1$ and $T 2$ transducers. The laser was turned off after $5 \mathrm{~s}$. Heating by the coated optical fiber tip is followed by both the generation of bubbles having different size and a change in the structure of the phantom, causing continuous changes in the oscillograms of the received ultrasound waves. To reduce the effect on the signal being studied by exposure to structural changes in the environment and sudden changes in differential signal amplitudes, it is reasonable to combine the method of signal processing including subtraction of the supporting signal with the approach of over-period subtraction. For this purpose, the supporting signal is continuously calculated as the average signal of the $N$ currently obtained oscillograms on the assumption that structural changes in the tissue will be insignificant over the period $N / f_{r}$. Thus, the signal obtained for study is the difference between the current oscillogram and the previous average value of the supporting signal. $N=3$ was used for this study. The signal processing results are shown in Fig. 5. After laser switching on the bubbles generation in the phantom (see Fig. 5(a)) is beginning. Those bubbles continue emerging after the laser switches off. The peak and average values of the signal spectra for noise $\left(t_{1}=3 \mathrm{~s}\right)$ and at the moment of laser switchoff $\left(t_{2}=10 \mathrm{~s}\right)$ are close to each other. In comparison with the bubble generation in water (Figs. 4(b) and 4(c)), the difference between the signal from the bubbles and the noise is reduced, and the signal spectrum is narrowed under the influence of changes in the viscosity of the phantom material. Study of

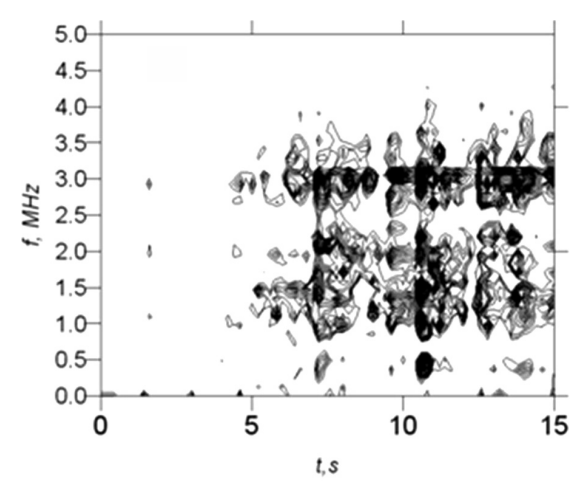

(a)

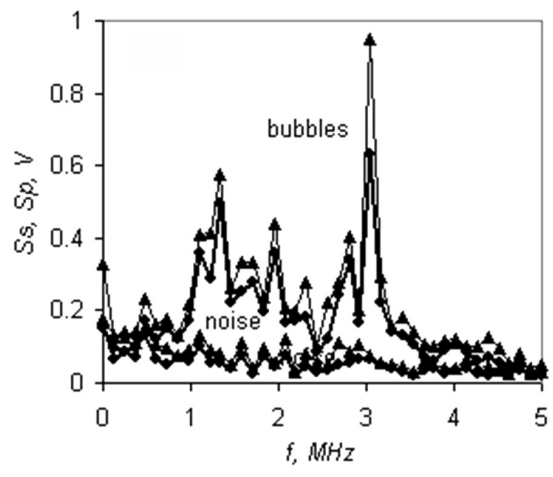

(b)

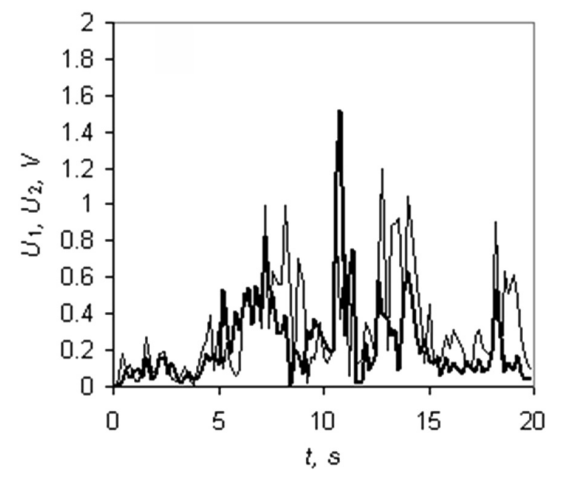

(c)

Fig. 5. Instantaneous signal (a) and current signal (b) in relation to the spectrum and the change of amplitude of the $f_{1}$ and $f_{2}$ frequencies with laser run-time. 
the harmonic amplitude changes for the $f_{1}$ and $f_{2}$ frequencies over time shows a periodicity of occurrence of harmonics, with the $0.15 \mathrm{~Hz}, 0.3 \mathrm{~Hz}$, and $0.9 \mathrm{~Hz}$ frequencies being caused by boiling in the water stream.

\section{Conclusion}

The studies conducted showed that an optical fiber with its tip shaded with a highly absorbing coating can be used as a source of simultaneous heating of biological tissues or fluids, and the generation of powerful micro-bubbles. Bubble generation depends on the power of the optical radiation and the absorption properties of the optical coating. In practice, during the destruction of the biological tissue the cutting conditions and the bubble generation intensity will be constantly changing. Bubble generation and control of this may be performed by means of pulse ultrasonic method using bubble excitation by simultaneous short and long pulses of ultrasonic waves. Conditions of bubble generation may be stabilized for the purpose of further use of detoxifying properties of bubbles in water or biological tissue. This stabilization may be achieved, for instance, by means of continuous monitoring of the bubble generation by determining the levels of the spectral components of the received ultrasonic wave signals. There are good reasons to use preliminary processing to increase the accuracy of detection of the signals from the bubbles. This preliminary processing consists of periodic subtraction of the average value of the oscillograms obtained during several previous ultrasound pulses from the currently received oscillogram of the signal.

\section{Acknowledgments}

The authors would like to acknowledge financial support from grant 14-15-00840 of the Russian Scientific Fund.

\section{References}

1. G. B. Altshuler, A. V. Belikov, Y. A. Sinelnik, "A laser-abrasive method for the cutting of enamel and dentine," Lasers Surg. Med. 28(5), 435-444 (2001).

2. G. E. Romanos, A. V. Belikov, A. V. Skrypnik, F. I. Feldchtein, M. Z. Smirnov, G. B. Altshuler,
"Uncovering dental implants using a new ThermoOptically Powered (TOP) technology with tissue air-cooling," Lasers Surg. Med. 47, 411-420 (2015).

3. J. G. Manni, Dental Applications of Advanced Lasers (DAAL), JGM Associates, US (2004).

4. Ch. Tarapacki, C. Kumaradas, R. Karshafian, "Enhancing laser thermal-therapy using ultrasound-microbubbles and gold nanorods of in vitro cells," Ultrasonics 53, 793-798 (2013).

5. G. E. Romanos, "Diode laser soft-tissue surgery: Advancements aimed at consistent cutting, improved clinical outcomes," Compend. Contin. Educ. Dent. (Jamesburg, N.J.) 34, 752-757 (1995).

6. D. S. Kuznetsova, M. M. Karabut, V. V. Elagin, M. A. Shakhova, V. I. Bredikhin, V. A. Kamensky, "Comparative analysis of biotissue laser resection using strongly absorbing optical fiber tips," Opt. Photon. J. 5(1) (2015), Available at http://dx.doi. org/10.4236/opj.2015.51001.

7. R. Mettin, "From a single bubble to bubble structures in acoustic cavitation, Oscillations, Waves and Interactions, T. Kurz, U. Parlitz, U. Kaatze, Eds., Universitatsverlag, Gottingen. pp. 171-198 (2007).

8. V. I. Yusupov, V. M. Chudnovskii, V. N. Bagratashvili, "Laser-induced hydrodynamics in watersaturated biotissues. 1. Generation of bubbles in liquid," Laser Phys. 20, 1641-1646 (2010).

9. T. Lifshitz, J. Levy, I. Klemperer, S. Levinger, "Anterior chamber gas bubbles after corneal flap creation with a femtosecond laser," J. Cataract. Refract. Surg. 31, 2227-2229 (2005).

10. B. Verhaagen, D. F. Rivas, "Measuring cavitation and its cleaning effect," Ultrasonics Sonochemistry 29, 619-628 (2016).

11. S. Chen, R. Kinnick, J. F. Greenleaf, M. Fatemi, "Difference frequency and its harmonic emitted by microbubbles under dual frequency excitation," Ultrasonics 44, e123-e126 (2006).

12. D. Z. Xi, Y. Gong, X. Gong, "Techniques to improve subharmonic emission from encapsulated microbubbles," AIP Conf. Proc. 1022, 464-467 (2008).

13. A. O. Maksimov, "On the subharmonic emission of gas bubbles under two-frequency excitation," Ultrasonics 35, 79-86 (1997).

14. T. G. Leighton, A. D. Phelps, D. G. Ramble, D. A. Sharpe, "Comparison of the abilities of eight acoustic techniques to detect and size a single bubble," Ultrasonics 34, 661-667 (1996).

15. A. J. Hardwick, "The mechanism of subharmonic ultrasound modulation by forcibly oscillated bubbles," Ultrasonics 33, 341-343 (1995).

16. T. Niederdrank, "Experimental investigations of the acoustic backscattering of a single cavitation bubble," Ultrasonics 35, 517-523 (1997). 
17. T. Christopher, "Source prebiasing for improved second harmonic bubble-response imaging," IEEE Trans. Ultrason. Ferroelectr. Freq. Control 46, 556-563 (1999).

18. T. Faez, M. Emmer, M. Docter, J. Sijl, M. Versluis, N. Jong, "Characterizing the subharmonic response of phospholipid-coated microbubbles for carotid imaging," Ultrasound Med. Biol. 37(6), 958-970 (2011).

19. K. Soetanto, M. Chan, "Study on the lifetime and attenuation properties of microbubbles coated with carboxylic acid salts," Ultrasonics 38, 969-977 (2000).

20. Y. Murata, Y. Watanabe, "Observation of vibration modes due to a small change in the initial radius of a microscopic bubble driven by intensive sound in water," Ultrasonics 34, 531-535 (1996).

21. H. J. Vos, Single Microbubble Imaging, Erasmus Medical Center. Rotterdam, Netherlands (2010).

22. A. D. Mansfel'd, D. A. Mansfel'd, A. M. Reyman, "Abilities of nonlinear acoustic methods in locating gas bubbles in biological tissues," Acoust. Phys. 51(2), 209-217 (2005).

23. A. D. Mansfel'd, G. P. Volkov, A. G. Sanin, I. A. Vladimirov, "Pulsed ultrasonic spectroscopy of gas bubbles," Acoust. Phys. 56(3), 290-298 (2010).

24. V. Daeichin, J. G. Bosch, A. Needles, "Subharmonic, non-linear fundamental and ultraharmonic imaging of microbubble contrast at high frequencies," Ultrasound Med. Biol. 41(2), 486-497 (2015).

25. Y. Zhang, X. Du, H. Xian, Y. Wu, "Instability of interfaces of gas bubbles in liquids under acoustic excitation with dual frequency," Ultrason. Sonochem. 23, 16-20 (2015).

26. Y. Zhang, S. Li, "Acoustical scattering cross section of gas bubbles under dual-frequency acoustic excitation," Ultrason. Sonochem. 26, 437-444 (2015).
27. C.-Y. Wu, M.-T. Lo, J. Tsa, D-H. Tsai, Y.-C. Chang, D.-R. Su, "The short-pulse subharmonic response of microbubbles based on a two-frequency approximation," Proc. IEEE Ultrasonics Symposium 2, pp. 1379-1382 (2004).

28. M.-T. Lo, J. Tsao, S. Lin, "Using the correlation property of subharmonic response as an index of cavitation of microbubbles," Proc. IEEE Ultrasonics Symposium 2, pp. 1383-1386 (2004).

29. J. Sijl, B. Dollet, M. Overvelde, V. Garbin, T. Rozendal, N. Jong, D. Lohse, M. Versluis, "Subharmonic behavior of phospholipid-coated ultrasound contrast agent microbubbles," J. Acoust. Soc. Am. 128(5), 3239-3252 (2010).

30. A. D. Phelps, T. G. Leighton, "High-resolution bubble sizing through detection of the subharmonic response with a two-frequency excitation technique," J. Acoust. Soc. Am. 99(4), 1985-1992 (1996).

31. Y. Zhang, "A generalized equation for scattering cross section of spherical gas bubbles oscillating in liquids under acoustic excitation," J. Fluids Eng. 135(9), 091301 (2013).

32. Y. Zhang, "Heat transfer across interfaces of oscillating gas bubbles in liquids under acoustic excitation," Int. Commun. Heat Mass Transf. 43, 1-7 (2013).

33. Y. Zhang, S. C. Li, "Notes on radial oscillations of gas bubbles in liquids: Thermal effects," J. Acoust. Soc. Am. 128(5), EL306-EL309 (2010).

34. M. Plesset, A. Prosperetti, "Bubble dynamics and cavitation," Ann. Rev. Fluid Mech. 9, 145-185 (1977).

35. M. A. Ainslie, T. G. Leighton, "Review of scattering and extinction cross-sections, damping factors, and resonance frequencies of a spherical gas bubble," J. Acoust. Soc. Am. 130(5), 3184-3208 (2011). 